\title{
Microstructural enrichment functions based on stochastic Wang tilings
}

\author{
Jan Novák \\ Institute of Structural Mechanics, Faculty of Civil Engineering, Brno University \\ of Technology, Veveři 95, 60200 Brno, Czech Republic \\ Department of Mechanics, Faculty of Civil Engineering, Czech Technical \\ University in Prague, Thákurova 7, 16629 Praha 6, Czech Republic \\ E-mail: novakj@cml.fsv.cvut.cz
}

\begin{abstract}
Anna Kučerová
Department of Mechanics, Faculty of Civil Engineering, Czech Technical University in Prague, Thákurova 7, 16629 Praha 6, Czech Republic

E-mail: anicka@cml.fsv.cvut.cz
\end{abstract}

\begin{abstract}
Jan Zeman
Department of Mechanics, Faculty of Civil Engineering, Czech Technical University in Prague, Thákurova 7, 16629 Praha 6, Czech Republic Centre of Excellence IT4Innovations, VŠB-TU Ostrava, 17. listopadu 15/2172, 70833 Ostrava-Poruba, Czech Republic

E-mail: zemanj@cml.fsv.cvut.cz
\end{abstract}

\begin{abstract}
This paper presents an approach to constructing microstructural enrichment functions to local fields in non-periodic heterogeneous materials with applications in Partition of Unity and Hybrid Finite Element schemes. It is based on a concept of aperiodic tilings by the Wang tiles, designed to produce microstructures morphologically similar to original media and enrichment functions that satisfy the underlying governing equations. An appealing feature of this approach is that the enrichment functions are defined only on a small set of square tiles and extended to larger domains by an inexpensive stochastic tiling algorithm in a non-periodic manner. Feasibility of the proposed methodology is demonstrated on constructions of stress enrichment functions for two-dimensional mono-disperse particulate media.
\end{abstract}


Keywords: Wang tiling, Microstructure optimisation, Enrichment functions, Partition of Unity, Trefftz method, FFT-based solver

\section{Introduction}

A detailed analysis of microstructured materials with the full resolution of heterogeneities by classical finite element methods has been found computationally prohibitive [34]. To overcome this, one option consists of modelling a coarse-scale problem with the help of homogenisation techniques based on effective material properties $[10,35,15]$. However, this may lead to a considerable loss of information on the fine scale behaviour, thereby resulting in an inaccurate assessment of microstructural effects on the global response and/or its evolution.

An alternative, computationally appealing, strategy proceeds from generalised finite element formulations that enhance the approximation properties of standard finite element spaces by subscale-informed enrichment functions. Their design involves two related but contradictory aspects: (i) realistic representation of the underlying heterogeneity patterns and (ii) construction of complex enrichment functions in a computationally efficient manner. Here, we briefly review these issues for two finite element frameworks. The first one is based on the partition of unity method, introduced by Melenk and Babuška [26] and generalised in numerous aspects later on $[2,13]$. The second one utilises the hybrid Trefftz stress formulations developed by Teixeira de Freitas [41], see also [17] for an overview. For simplicity, we restrict our attention to the small-strain linear elasticity in two dimensions. The following nomenclature is used in the sequel. Scalar quantities are denoted by plain letters, e.g. $a$ or $A$, vectors and matrices are in bold as, e.g. $\boldsymbol{a}$ or $\boldsymbol{A}$. In addition, we adopt the Mandel vector-matrix representation of symmetric second- and fourthorder tensors, e.g. $a_{i j}$ or $A_{i j k l}$, so that [28, Section 2.3]

$$
\boldsymbol{a}=\left[\begin{array}{c}
a_{11} \\
a_{22} \\
\sqrt{2} a_{12}
\end{array}\right], \quad \boldsymbol{A}=\left[\begin{array}{ccc}
A_{1111} & A_{1122} & \sqrt{2} A_{1112} \\
A_{2211} & A_{2222} & \sqrt{2} A_{2212} \\
\sqrt{2} A_{1211} & \sqrt{2} A_{1222} & 2 A_{1212}
\end{array}\right] .
$$

\subsection{Partition of unity methods}

Consider a microstructured two-dimensional domain $\mathcal{O} \subset \mathbb{R}^{2}$ approximated by finite elements, cf. Figure 1. The partition of unity methods build on the displacement 


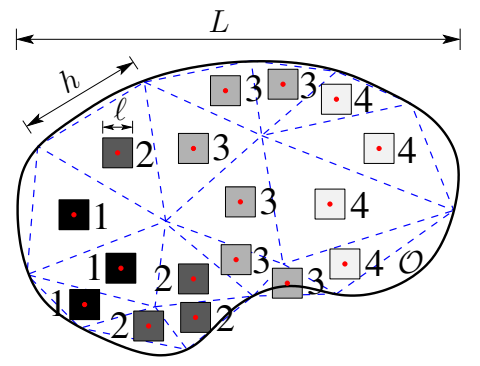

(a)

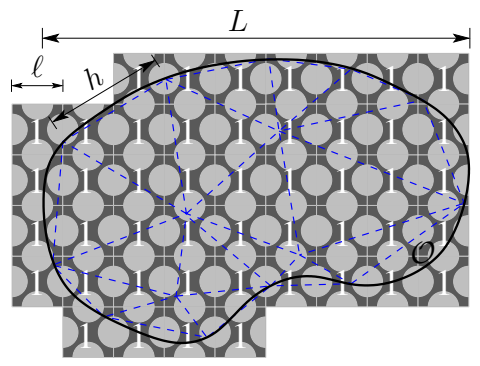

(b)

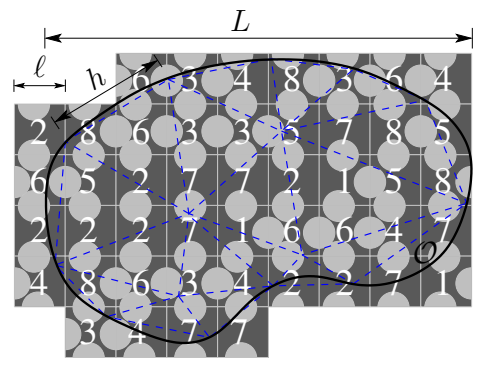

(c)

Figure 1. Examples of heterogeneity representations for a macroscopic body $\mathcal{O}$ and (a) separated scales $(\ell \ll h<L)$ : three unit cells associated with integration points (in red), (b) periodic geometry with non-separated scales $(\ell<h<L$ ): a single periodic unit cell, (c) aperiodic geometry with non-separated scales $(\ell<$ $h<L)$ : eight distinct Wang tiles; $\ell, h$, and $L$ denote characteristic macroscopic, element (meso-scopic), and heterogeneity lengths, respectively.

field approximation in the form

$$
\boldsymbol{u}(\boldsymbol{x}) \approx \sum_{n=1}^{n^{\mathrm{n}}} N_{n}(\boldsymbol{x})\left[\boldsymbol{a}_{n}+\Psi^{*}(\boldsymbol{x}) \boldsymbol{b}_{n}\right] \text { for } \boldsymbol{x} \in \mathcal{O}
$$

where $n^{\mathrm{n}}$ is the number of nodes in the finite element mesh, $N_{n}: \mathcal{O} \rightarrow \mathbb{R}$ denotes the standard finite element basis functions and $\boldsymbol{a}_{n} \in \mathbb{R}^{2}$ the regular degrees of freedom associated with the $n$-th node, whereas $\boldsymbol{\Psi}^{*}$ and $\boldsymbol{b}_{n}$ designate the strategy-specific matrices of enrichment functions and extended degrees of freedom, respectively. The ansatz (1) is then employed in the standard Galerkin procedure to arrive at a system of linear(ized) equations involving both regular and extended degrees of freedom. This approach was explored by Fish and Yuan [11, 12], who derived the enrichment functions from solutions to a periodic unit cell problem, formulated for cells associated with integration points, see Figure 1(a). In particular,

$$
\boldsymbol{\Psi}^{*}(\boldsymbol{x})=\left[\begin{array}{lll}
u_{1}^{*(1)} & u_{1}^{*(2)} & \frac{1}{\sqrt{2}} u_{1}^{*(3)} \\
u_{2}^{*(1)} & u_{2}^{*(2)} & \frac{1}{\sqrt{2}} u_{2}^{*(3)}
\end{array}\right](\boldsymbol{x}),
$$

where $u_{i}^{*(j)}: \mathcal{O} \rightarrow \mathbb{R}$ denotes the $i$-th component of the fluctuating displacement field, determined for a unit cell subject to the average strain with the $j$-th component set to one, while the remaining two vanish (see Appendix A for further details). Such 
form of enrichment functions is motivated by the displacement decomposition

$$
\boldsymbol{u}(\boldsymbol{x})=\boldsymbol{u}^{0}(\boldsymbol{x})+\boldsymbol{u}^{*}(\boldsymbol{x}) \text { for } \boldsymbol{x} \in \mathcal{O},
$$

with $\boldsymbol{u}^{0}: \mathcal{O} \rightarrow \mathbb{R}^{2}$ and $\boldsymbol{u}^{*}: \mathcal{O} \rightarrow \mathbb{R}^{2}$ referring to global and fluctuating displacement fields; parameter $\boldsymbol{b}_{n} \in \mathbb{R}^{3}$ in Equation (1) has thus the physical meaning of a generalised average strain known from classical homogenisation theories [15]. Since such fields are constructed under the assumption of separated lenghtscales, Figure $1(\mathrm{a})$ with $\ell / L \rightarrow 0$, an attention is paid neither to the geometrical compatibility among neighbouring cells, nor to the compatibility of the corresponding enrichment fields. Consistent mathematical results for periodic media with a finite ratio $\ell / L$, Figure 1(b), were obtained by Matache et al [25]. The enrichment functions are constructed on the basis of the spectral version of the unit cell problem [29] resolved by the $p$-version of the finite element method, see [1] for additional contributions to this field.

The partition of unity methods have also been applied to simulations of material systems with explicitly represented non-periodic heterogeneities, such as thin fibres [37, 38]. Here, the enrichment function is chosen to be piecewise constant in fibre and matrix domains, and the extended degrees of freedom correspond to a relative slip at the fibre-matrix interface. Such simple format comes at the expense of the fact that two extra degrees of freedom are introduced per fibre, which renders realistic simulations costly.

\subsection{Trefftz method}

The hybrid Trettfz approach has recently been employed by Novák et al [32] to simulate composites reinforced with non-periodic ellipsoidal heterogeneities with nonseparated lengthscales. The method builds on the additive stress decomposition

$$
\boldsymbol{\sigma}(\boldsymbol{x})=\boldsymbol{\sigma}^{0}(\boldsymbol{x})+\boldsymbol{\sigma}^{*}(\boldsymbol{x}) \text { for } \boldsymbol{x} \in \mathcal{O},
$$

with $\boldsymbol{\sigma}^{0}: \mathcal{O} \rightarrow \mathbb{R}^{3}$ corresponding to the macroscopic stress field and $\boldsymbol{\sigma}^{*}: \mathcal{O} \rightarrow \mathbb{R}^{3}$ being stress fluctuations, approximated at the level of an element $\Omega_{e}$ as

$$
\boldsymbol{\sigma}(\boldsymbol{x}) \approx \boldsymbol{\Sigma}_{e}(\boldsymbol{x}) \boldsymbol{a}_{e}+\boldsymbol{\Sigma}^{*}(\boldsymbol{x}) \boldsymbol{b}_{e} \text { for } \boldsymbol{x} \in \Omega_{e} .
$$

Here, in analogy to Equation (1), $\boldsymbol{\Sigma}_{e}: \Omega_{e} \rightarrow \mathbb{R}^{3 \times m}$ stands for the standard basis functions of the Trefftz method associated with $m$ regular degrees of freedom $\boldsymbol{a}_{e} \in R^{m}$ 
and $\boldsymbol{b}_{e} \in \mathbb{R}^{3}$ denotes the extended degrees of freedom with the physical meaning of average element strains. The individual enrichment functions

$$
\boldsymbol{\Sigma}^{*}(\boldsymbol{x})=\left[\begin{array}{ccc}
\sigma_{11}^{*(1)} & \sigma_{11}^{*(2)} & \sqrt{2} \sigma_{11}^{*(3)} \\
\sigma_{22}^{*(1)} & \sigma_{22}^{*(2)} & \sqrt{2} \sigma_{22}^{*(3)} \\
\sqrt{2} \sigma_{12}^{*(1)} & \sqrt{2} \sigma_{12}^{*(2)} & 2 \sigma_{12}^{*(3)}
\end{array}\right](\boldsymbol{x}) \text { for } \boldsymbol{x} \in \mathcal{O},
$$

correspond to the fluctuating stress fields due to unitary strain impulses, see again Appendix A for further details. Note that the regular and enrichment basis functions need to be selected such that the stress remains self-equilibrated. The stress approximation is complemented with an independent approximation of displacements at the element boundary $\Gamma_{e}[18]$

$$
\boldsymbol{u}(\boldsymbol{x}) \approx \boldsymbol{N}_{e}^{\Gamma}(\boldsymbol{x}) \boldsymbol{a}_{e}^{\Gamma} \text { for } \boldsymbol{x} \in \Gamma_{e},
$$

involving only regular edge shape functions $\boldsymbol{N}_{e}^{\Gamma}$ and regular boundary degrees of freedom $\boldsymbol{a}_{e}^{\Gamma}$. The remainder of the formulation follows from the weak form of the equilibrium and compatibility equations, which can be converted to the element boundaries by virtue of the divergence theorem, cf. [18, 41]. The appealing feature of the particular formulation [32] is that the size of the resulting system of equations is the same as for the homogeneous problem, due to the elimination of the extended degrees of freedom. This is achieved by a careful construction of the enrichment functions through Eshelby solutions for individual particles [8, 9], combined together to obtain compatible mechanical fields [32].

\subsection{Tiling-based approach}

This short overview illustrates the major difficulty in simulating non-periodic systems with realistic geometries, namely that simple enrichment functions lead to the loss of information and/or to a significant increase in the number of degrees of freedom, whereas manageable system sizes necessitate complex constructions of enrichment functions. The aim of this work is thus to develop an algorithm that allows for extending the local (possibly periodic) data from computationally tractable samples to entire macroscopic domains in a non-periodic way, Figure 1(c). The algorithm keeps the synthesised enrichment functions, $\boldsymbol{\Psi}^{*}$ in Equation (1) or $\boldsymbol{\Sigma}^{*}$ in Equation (5), continuous across congruent boundaries and consistent in terms of statistical properties of original and reconstructed material morphologies. It is based on a 
small number of the so-called Wang tiles [44, 16, 4] and a stochastic tiling procedure introduced by Cohen et al. [3].

In 1961, Hao Wang introduced a tiling concept involving square tiles with different codes on their edges, referred to as Wang tiles [44]. The tiles are connected together so that the adjacent edges have the same code and permit a computationally efficient graphic reproduction of morphological patterns $[3,4,6,16]$. Their desirable aesthetic properties are attributed to the aperiodicity of tilings, whereas the low computational effort results from the use of a small number of tiles to compress the entire morphological information [21].

Here, we exploit and extend these principles to provide a basis for an efficient generation of microstructure-based enrichment functions applicable in partition of unity or hybrid Trefftz finite element algorithms. In order to meet additional criteria arising from such constructions, the Simulated annealing-based optimisation [19, 43] is used to arrive at optimal tile sets. The performance of the method is illustrated on the construction of tile-based stress enrichment functions in a mono-disperse twophase composite medium with linear elastic phases. Although the proposed approach is illustrated solely in the two-dimensional setting, it is fully extensible to three dimensions by exploring the results available for the Wang cubes [5, 24]. We also note in passing that the techniques developed in this paper can be used equally well as microstructure reconstruction or generation algorithms, generalising the previous developments available e.g. in [36, 46, 20, 48, 22, 31, 40].

The paper structure is as follows. The concept of stochastic Wang tiling is described in Section 2. A discussion on the optimisation procedure based on prescribed statistical descriptors and compatibility of synthesised mechanical fields on contiguous tile edges is given in Section 3. Section 4 comprises numerical examples demonstrating the performance of the proposed approach. Final remarks on the current developments and future plans are assembled in Section 5. Finally, in Appendix A, we present a brief overview of the stress analysis algorithm utilised to determine the local stress fluctuations.

\section{Aperiodic tilings by sets of Wang tiles}

Consider again the domain $\mathcal{O}$ from Figure 1(c) covered by a regular square grid. Each grid cell contains specific microstructural patterns that are compatible on contiguous boundaries. If there are no missing cells inside the synthesised domain, 
the discretization is called a valid tiling $\ddagger$ and a single cell is referred to as the Wang tile [44], Figure 2. The tiles have different codes on their edges, enumerated here by lowercase Greek letters, and are not allowed to rotate during the tiling procedure. The number of distinct tiles is fixed, though arranged in such a fashion that no sub-sequence of tiles periodically repeats. The set of all distinct tiles is referred to as the tile set, Figure 2(a). Sets that enable uncountably many, always aperiodic, tilings are called aperiodic sets [4]. The assumption of strictly aperiodic sets can be relaxed, though still being capable to tile the plane aperiodically, e.g., when utilising the Cohen-Shade-Hiller-Deussen (CSHD) tiling algorithm [3] briefly introduced in the following section. Note that such tilings provide substantial generalisations to periodic paving algorithms, which use identical tiles-periodic unit cells, recall Figure 1(b).

(a)
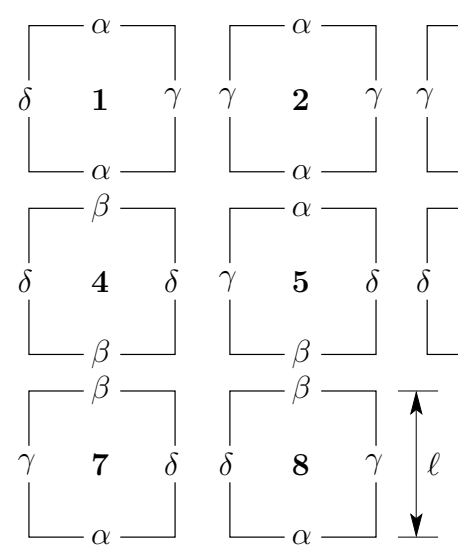
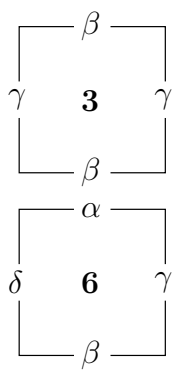

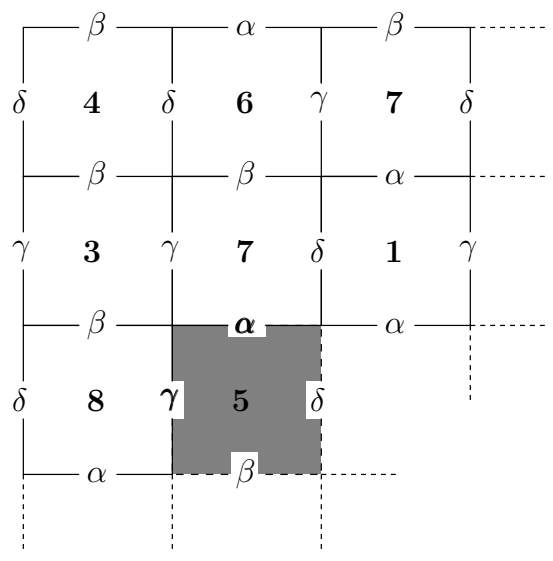

Figure 2. (a) Tile set W8/2-2 [3] consisting of 8 tiles with 2 vertical $\{\alpha, \gamma\}$ and 2 horizontal $\{\beta, \delta\}$ edge codes with equal frequencies of occurrence $q_{\alpha}=q_{\beta}=q_{\gamma}=$ $q_{\delta}=\frac{1}{4}$, and $n^{\mathrm{NW}}=2$, (b) an example of aperiodic valid tiling with highlighted connectivity across south-eastern and north-western edges.

\subsection{Tile set setup}

Favourable properties of a tile set to control repetitive effects proceed from the tile and edge code diversity. The number of edge codes $n_{i}^{\mathrm{c}}$ in the $i$-th spatial direction $\ddagger$ Henceforth, the term "tiling" stands for "valid tiling" exclusively, thereby excluding invalid tilings from the consideration. 
of the Cartesian coordinates can be chosen arbitrarily, while the number of tiles $n^{\mathrm{t}}$ must satisfy

$$
n^{\mathrm{t}}=n^{\mathrm{NW}} \sqrt{n^{\mathrm{cs}}}
$$

where $n^{\mathrm{cs}}=\left(n_{1}^{\mathrm{c}} n_{2}^{\mathrm{c}}\right)^{2}$ is the number of tiles in the complete set and $n^{\mathrm{NW}}=2, \ldots, \sqrt{n^{\mathrm{cs}}}$ stands for the number of tiles associated with each admissible pair of northwestern (NW) edge codes, Figure 2(a), see [33] for further details.

When designing a tile set, one chooses a particular number of edge codes $n_{1}^{\mathrm{c}}$ and $n_{2}^{\mathrm{c}}$. The complete set of $n^{\mathrm{cs}}$ tiles is created by mutually permuting the codes. In order to tile the plane, the south-eastern edge codes must match those assigned to NW edges, Figure 2(b). Thus, the created tiles are collected according to NW combinations. Finally, a desired number of tiles is chosen using Equation (8), in such a way that $n^{\mathrm{NW}}$ unique tiles is selected from each NW group. The emerging, userdefined, set of tiles is referred to as $\mathrm{W} n^{\mathrm{t}} / n_{1}^{\mathrm{c}}-n_{2}^{\mathrm{c}}$. Moreover, we denote the relative frequency of occurrence of the $c$-th code in the tile set by $q_{c}$, see Figure 2(a).

\subsection{CSHD stochastic tiling algorithm}

Since there are $n^{\mathrm{NW}}$ tiles associated to each NW group, index of the new tile to be placed is selected randomly from the set $\left\{1, \ldots, n^{\mathrm{NW}}\right\}$ with the uniform probability. Beforehand, one must select an appropriate NW group compatible with the eastern code of a previously placed tile and the southern code of the tile just above the one to be placed (edges $\alpha$ and $\gamma$ of shaded areas in Figure 2(b)). Aperiodicity of the resulting tiling is guaranteed by assuming that the random generator never returns a periodic sequence of numbers and that each NW group contains at least two distinct tiles [3].

\section{Designing optimal tile set morphology}

To simplify the exposition, we limit our attention to two-phase composite media formed by a matrix phase and equi-sized disks of radius $\rho$ and a parametric microstructure representation built on the Wang tile set W8/2-2 $\S$, introduced in Section 3.1. The location of the disks within the tiles has to be optimised to

$\S$ The set W8/2-2 has been chosen since it is the simplest one that allows for aperiodic patterns in the stochastic sense [3]. Note that all the steps of the tile set design can be directly generalised to more complex tile sets, cf. [33]. 
achieve (i) good approximation of the original microstructure in terms of a given morphological descriptor, Section 3.2, and (ii) microstructures that guarantee the compatibility of enrichment functions on contiguous tile edges, Section 3.3. Such criteria originate from different perspectives. The first goal aims at capturing the dominant spatial features of original media, while the latter criterion ensures that the tiling-generated fields comply with the governing differential equations. The details of the algorithm used to solve the resulting optimisation problem are provided in Section 3.4.

\subsection{Microstructure parametrisation}

The adopted bitmap-based microstructure representation involves a Wang tile set consisting of $n^{\mathrm{t}}$ tiles of the edge length $\ell \in \mathbb{N}$ (in pixels), in which we distribute $n^{\mathrm{d}}$ disks of radius $\rho$. The $d$-th disk is represented by a triplet $\left\{t_{d}, x_{1, d}, x_{2, d}\right\}$, where $t_{d} \in\left\{1, \ldots, n^{\mathrm{t}}\right\}$ denotes the tile index and $x_{d, j} \in\{1, \ldots, \ell\}$ specifies the position of the $d$-th disk within the tile at the $j$-th direction. The associated parameter vector $\boldsymbol{p}$ is obtained as a collection of these data:

$$
\boldsymbol{p}=\left[t_{d}, x_{1, d}, x_{2, d}\right]_{d=1}^{n^{\mathrm{d}}} .
$$

Since the position of each disk is specified by three parameters, the parameter space $\mathbb{P}$ is $\left(3 \times n^{\mathrm{d}}\right)$-dimensional, i.e. $\mathbb{P} \subset \mathbb{N}^{3 \times n^{\mathrm{d}}}$.

In an admissible configuration, the disks do not penetrate each other or overlap corners of tiles being associated with. The first constraint reflects the given feature of the original microstructure, Figure 3(a), whereas the latter one arises as an artifact intrinsic to the edge-based tiling algorithm, e.g., [3]. In addition, to maintain the morphological compatibility, any disk intersecting the edge of a given code needs also be associated to tiles containing the same edge. To emphasise this, we encode a particular microstructural configuration as $n^{\mathrm{d}}\left\{n_{c}^{\mathrm{d}}\right\}_{c=1}^{n^{\mathrm{c}}}$, where $n_{c}^{\mathrm{d}}$ denotes the number of disks intersecting the edge of code $c$, see Figure 6 on page 15.

\subsection{Statistical properties of the microstructure}

The most common class of statistical descriptors embodies a set of $n$-point probability functions, applicable to generic heterogeneous media [42]. In this paper, the focus

is on the two-point probability function, which captures primary phenomena as 
the phase volume fraction, characteristic microstructural length(s), and long-range orientation orders, if any.

We now assume that the domain $\mathcal{O}$ is occupied by a two-phase heterogeneous material discretized by a regular lattice of $n_{1}^{\mathcal{O}} \times n_{2}^{\mathcal{O}}$ pixels, indexed by $\boldsymbol{k} \in \mathbb{K}^{\mathcal{O}}$ with

$$
\mathbb{K}^{\mathcal{O}}=\left\{\boldsymbol{m} \in \mathbb{Z}^{2}:-\frac{n_{i}^{\mathcal{O}}}{2}<m_{i} \leq \frac{n_{i}^{\mathcal{O}}}{2}, i=1,2\right\} .
$$

The distribution of individual phases (disks and matrix) within $\mathcal{O}$ is quantified by the characteristic function $\chi(\boldsymbol{k})$, which equals 1 when $\boldsymbol{k}$ is occupied by the disk phase and 0 otherwise, cf. Figure 3(a). Assuming a periodic $\|$ ergodic medium, the two-point probability function $S_{2}: \mathbb{K}^{\mathcal{O}} \rightarrow[0,1]$ is then defined as [42]

$$
S_{2}(\boldsymbol{k})=\frac{1}{n_{1}^{\mathcal{O}} n_{2}^{\mathcal{O}}} \sum_{\boldsymbol{m} \in \mathbb{K}^{\mathcal{O}}} \chi(\boldsymbol{m}) \chi\left(\lfloor\boldsymbol{k}+\boldsymbol{m}\rfloor_{\mathbb{K}^{\mathcal{O}}}\right),
$$

where $\lfloor\bullet\rfloor_{\mathbb{K}_{\mathcal{O}}}$ denotes the $\mathbb{K}^{\mathcal{O}}$-periodic extension. Noticing that (11) has the structure of circular correlation, the two-point probability function can be efficiently evaluated using Fast Fourier Transform techniques, see e.g. [14].

According to its definition, $S_{2}(\boldsymbol{k})$ quantifies the probability that two arbitrary points separated by $\boldsymbol{k}$ will both be located at the disk phase when randomly selected from $\mathbb{K}^{\mathcal{O}}$. Denoting by $\phi$ the disk volume fraction, $0 \leq \phi \leq 1$, the two-point probability function satisfies $S_{2}(\mathbf{0})=\phi$. Moreover, $S_{2}(\boldsymbol{k}) \simeq \phi^{2}$ for $\|\boldsymbol{k}\| \gg \rho$ indicates that the medium does not exhibit repeating long-range order orientation effects, cf. Figure 3(b).

The following procedure is adopted to determine the two-point probability function for the tile-based microstructure. First, the set W8/2-2 is used to assemble a $4 \times 4$ tiling $\mathcal{O}_{\mathrm{S}} \subset \mathbb{R}^{2}$, periodic on external boundaries, in which each tile appears with the same frequency in order to suppress artificial fluctuations in volume fractions, Figure 4(a). The domain $\mathcal{O}_{\mathrm{S}}$ is discretized by an $n_{1}^{\mathcal{O}_{\mathrm{S}}} \times n_{2}^{\mathcal{O}_{\mathrm{S}}}$ regular grid with the same pixel size as in the original microstructure, so that $n_{i}^{\mathcal{O}_{\mathrm{S}}}<n_{i}^{\mathcal{O}}$. Given a parameter vector $\boldsymbol{p}$ quantifying positions of individual disks, the tile-based morphology is quantified by the two-point probability function $\widetilde{S}_{2}: \mathbb{P} \times \mathbb{K}^{\mathcal{O}_{\mathrm{S}}} \rightarrow[0,1]$, and its \| Note that periodicity is considered here for the sake of computational efficiency. The tilinggenerated data is always aperiodic. 
(a)

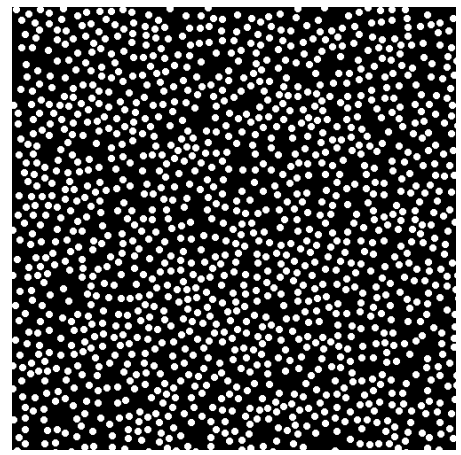

Figure 3. (a) An example of two-phase medium formed by equilibrium distribution of 1,300 equi-sized disks of volume fraction $26.8 \%$ and (b) the twopoint probability function $S_{2}$; the sample is discretized with $1,000 \times 1,000$ pixels and each disk has the radius of 8 pixels.

proximity to the target microstructure is evaluated as

$$
f^{\mathrm{S}}(\boldsymbol{p})=\frac{1}{n_{1}^{\mathcal{O}_{\mathrm{S}}} n_{2}^{\mathcal{O}_{\mathrm{S}}}} \sum_{\boldsymbol{k} \in \mathbb{K}^{\mathcal{O}_{\mathrm{S}}}}\left(S_{2}(\boldsymbol{k})-\widetilde{S}_{2}(\boldsymbol{p}, \boldsymbol{k})\right)^{2},
$$

where $\mathbb{K}^{\mathcal{O}_{\mathrm{S}}}$ is defined analogously as for the target medium $\mathcal{O}$.

\begin{tabular}{|l|l|l|l|}
\hline 3 & 4 & 1 & 6 \\
\hline 5 & 7 & 2 & 8 \\
\hline 2 & 1 & 4 & 7 \\
\hline 8 & 6 & 3 & 5 \\
\hline
\end{tabular}

(a) Tiling $\mathcal{O}_{\mathrm{S}}$

\begin{tabular}{||l||l|l|l|l||l|l|l|l||}
\hline 2 & 1 & 6 & 3 & 4 & 8 & 3 & 6 & 4 \\
\hline 2 & 8 & 6 & 3 & 3 & 5 & 7 & 8 & 5 \\
\hline 6 & 5 & 2 & 7 & 7 & 2 & 1 & 5 & 8 \\
\hline 2 & 2 & 2 & 7 & 1 & 6 & 6 & 4 & 7 \\
\hline 4 & 8 & 6 & 3 & 4 & 2 & 2 & 7 & 1 \\
\hline 3 & 3 & 4 & 7 & 7 & 2 & 8 & 5 & 8 \\
\hline 1 & 5 & 7 & 1 & 3 & 4 & 1 & 6 & 5 \\
\hline 8 & 6 & 5 & 8 & 5 & 1 & 4 & 8 & 4 \\
\hline 1 & 4 & 2 & 7 & 2 & 2 & 7 & 1 & 5 \\
\hline
\end{tabular}

(b) Tiling $\mathcal{O}_{\mathrm{T}}$

Figure 4. Valid tilings used in optimisation with respect to (a) two-point probability function and (b) stress field; highlighted vertical edges in (b) correspond to edge set $\Gamma_{\delta}$ containing 50 equivalent edges of code $\delta$ and length $\ell$. Tiles denoted by bold numbers (first two rows) are used to generate aperiodic enrichment functions. 


\subsection{Stress-based enrichment fields and their compatibility}

The additional, yet more complex, goal is to find the tile set morphology that ensures the admissibility of enrichment functions synthesised by the tiling algorithm. Analogously to the original Wang idea, this is achieved by requiring that edges of identical codes carry identical, this time non-scalar, information. In particular, motivated by encouraging results obtained recently in [32], we concentrate on the stress enrichment functions $\Sigma^{*}$, recall Equation (6). It is natural convert them to equivalent traction fluctuations, obtained as

$$
T^{*}=\nu \Sigma^{*}
$$

where $\boldsymbol{T}^{*}$ collects the components associated with individual load-cases and $\boldsymbol{\nu}$ stores the components of the normal vector:

$$
\boldsymbol{T}^{*}=\left[\begin{array}{ccc}
T_{1}^{*(1)} & T_{1}^{*(2)} & T_{1}^{*(3)} \\
T_{2}^{*(1)} & T_{2}^{*(2)} & T_{2}^{*(3)}
\end{array}\right], \quad \boldsymbol{\nu}=\left[\begin{array}{ccc}
\nu_{1} & 0 & \frac{1}{\sqrt{2}} \nu_{2} \\
0 & \nu_{2} & \frac{1}{\sqrt{2}} \nu_{1}
\end{array}\right] .
$$

Analogously to the morphology design, the definition of the traction-based objective function is based on an auxiliary $9 \times 9$ tiling $\mathcal{O}_{\mathrm{T}}$, Figure $4(\mathrm{~b})$, discretized into $n_{1}^{\mathcal{O}_{\mathrm{T}}} \times n_{2}^{\mathcal{O}_{\mathrm{T}}}$ bitmap with pixels indexed by $\boldsymbol{k} \in \mathbb{K}^{\mathcal{O}_{\mathrm{T}}}$. The tiling is periodic at external boundaries, and contains all admissible combinations of tile pairs from the set W8/2-2 sharing all edge codes $\Phi$, since we assume that the edge traction values are dominated by the response of adjacent tiles. Hence, for each edge code $c \in\left\{1,2, \ldots, n^{\mathrm{c}}\right\}$ with $n^{\mathrm{c}}=n_{1}^{\mathrm{c}}+n_{2}^{\mathrm{c}}$, we introduce a set $\Gamma_{c}$ formed by $n^{\Gamma_{c}}$ edges of identical code $c$ and length $\ell$ with normal vector $\boldsymbol{\nu}^{\Gamma_{c}}$, Figure $4(\mathrm{~b})^{+}$. By $\boldsymbol{\Gamma}_{c, j}:\{1, \ldots, \ell\} \rightarrow \mathbb{K}^{\mathcal{O}_{\mathrm{T}}}, j \in\left\{1,2, \ldots, n^{\Gamma_{c}}\right\}$, we denote a function providing coordinates of individual pixels at the $j$-th edge of code $c$.

Now we are in a position to quantify differences of tractions carried by an edge code $c$, due to differing neighbours, via an objective function $f_{c}^{\mathrm{T}}$. For a given parameter vector $\boldsymbol{p} \in \mathbb{P}$ and material properties of individual phases, we calculate the stress enrichment function $\boldsymbol{\Sigma}^{*}(\boldsymbol{p}, \boldsymbol{k})$ by the algorithm outlined in Appendix A,

ฯ There are 16 distinct pair combinations of basic tiles sharing the code $\delta$ : $\{2-1,2-2,2-7,2-$ $8,4-1,4-2,4-7,4-8,5-1,5-2,5-7,5-8,7-1,7-2,7-7,7-8\}$ see Figure $2(\mathrm{a})$. All these combinations are present in the tiling $\mathcal{O}_{\mathrm{T}}$ in Figure $4(\mathrm{~b})$, each of them multiple times.

+ For the particular tile set considered here, we set $\boldsymbol{\nu}^{\Gamma_{\alpha}}=\boldsymbol{\nu}^{\Gamma_{\gamma}}=[0,1]$ and $\boldsymbol{\nu}^{\Gamma_{\beta}}=\boldsymbol{\nu}^{\Gamma_{\delta}}=[1,0]$. 
and evaluate the objective function as

$$
f_{c}^{\mathrm{T}}(\boldsymbol{p})=\frac{1}{\ell} \sum_{s=1}^{\ell}\left\|\max \left\{\boldsymbol{T}^{*}\left(\boldsymbol{p}, \boldsymbol{\Gamma}_{c, j}(s)\right)\right\}_{j=1}^{n^{\Gamma_{c}}}-\min \left\{\boldsymbol{T}^{*}\left(\boldsymbol{p}, \boldsymbol{\Gamma}_{c, j}(s)\right)\right\}_{j=1}^{n^{\Gamma_{c}}}\right\|_{1},
$$

where the traction enrichments are determined from Equation (13) with $\boldsymbol{\nu}=\boldsymbol{\nu}^{\Gamma_{c}}$, $\max$ and min operations are understood component-wise and $\|\boldsymbol{A}\|_{1}=\sum_{i, j}\left|A_{i j}\right|$. Collecting the contributions from all codes, we obtain

$$
f^{\mathrm{T}}(\boldsymbol{p})=\sum_{c=1}^{n^{\mathrm{c}}} f_{c}^{\mathrm{T}}(\boldsymbol{p})
$$

Once the tile set is designed with respect to the objective function (15), the tiling-based stress enrichment functions $\widetilde{\boldsymbol{\Sigma}}^{*}: \mathbb{K}^{\mathcal{O}_{\mathrm{T}}} \rightarrow \mathbb{R}^{3 \times 3}$ can be assembled by the CSHD algorithm using the stress fluctuations $\Sigma^{*}$ carried by an arbitrary selection of tiles 1-8 from the tiling $\mathcal{O}_{\mathrm{T}}$. In the numerical experiments reported in Section 4 , we use the set of eight tiles from the top rows of $\mathcal{O}_{\mathrm{T}}$ highlighted by bold numbers in Figure 4(b), but equivalent results were obtained for different selections. Due to this procedure, the reconstructed edge tractions corresponding to the synthesised enrichments $\widetilde{\boldsymbol{\Sigma}}^{*}$ may experience jumps at tile edges. For the $j$-th edge of the set $\Gamma_{c}$, these are defined as

$$
\left[\widetilde{\boldsymbol{T}}_{c, j}^{*}\right](s)=\boldsymbol{\nu}^{\Gamma_{c}}\left(\widetilde{\boldsymbol{\Sigma}}_{+}^{*}\left(\boldsymbol{\Gamma}_{c, j}\right)-\widetilde{\boldsymbol{\Sigma}}_{-}^{*}\left(\boldsymbol{\Gamma}_{c, j}\right)\right) \text { for } s \in\{1,2, \ldots, \ell\},
$$

where $\widetilde{\boldsymbol{\Sigma}}_{+}^{*}$ and $\widetilde{\boldsymbol{\Sigma}}_{-}^{*}$ denote the values of the stress enrichment functions taken from the nearest edge neighbours from right and left, respectively, relative to the orientation of the edge set $\Gamma_{c}$ by the normal vector $\boldsymbol{\nu}^{\Gamma_{c}}$.

\subsection{Optimisation procedure}

In fact, the goals represented by objective functions (12) and (15) are conflicting. Minimising only with respect to the two-point probability function results in traction enrichments discontinuous at internal edges, whereas the latter criterion drives the system to a periodic distribution of disks. To achieve a compromise solution, we introduce a composite objective function in the form

$$
f(\boldsymbol{p})=w f^{\mathrm{S}}(\boldsymbol{p})+f^{\mathrm{T}}(\boldsymbol{p}),
$$


where $w$ denotes a weighting factor balancing geometrical features with mechanical compatibility. The minimisation of the objective function (17) is performed by the well-established Simulated Annealing method [19, 43], extended by a re-annealing phase to escape from local extremes, e.g. [23].

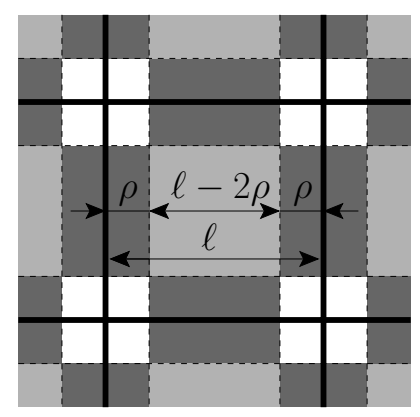

Figure 5. Tile decomposition into interiors, edges and corner regions.

Given the number of disks $n^{\mathrm{d}}$ and the target volume fraction $\phi$, we initiate the algorithm by determining the number of edge disks $n_{c}^{\mathrm{d}}$ related to the $c$-th code and the tile edge length $\ell$. Although this problem is difficult due to multiplicity of the edge-related disks, recall Figure 6, we resolved it by a heuristic procedure outlined next. To this purpose, an arbitrary tile is decomposed into three regions assigned to interiors (light grey area in Figure 5), edges (dark grey area in Figure 5), and to corners (white area in Figure 5 that cannot be occupied by disks due to the corner constraint). For a disk configuration $n^{\mathrm{d}}\left\{n_{c}^{\mathrm{d}}\right\}_{c=1}^{n^{\mathrm{c}}}$ related to a tile set $\mathrm{W} n^{\mathrm{t}} / n_{1}^{\mathrm{c}}-n_{2}^{\mathrm{c}}$, there is $\left(n^{\mathrm{d}}-\sum_{c=1}^{n^{\mathrm{c}}} n_{c}^{\mathrm{d}}\right)$ interior disks and, due to the edge constraints, a single disk associated with code $c$ appears $2 n^{t} q_{c}$ times, cf. Figure 6 . Thus, the disk volume fraction in the tile set or in a tiling is given by

$$
\widetilde{\phi} \approx \frac{A^{\mathrm{d}}}{n^{\mathrm{t}} \ell^{2}}\left(n^{\mathrm{d}}+\sum_{c=1}^{n^{\mathrm{c}}}\left(2 n^{\mathrm{t}} q_{c}-1\right) n_{c}^{\mathrm{d}}\right),
$$

with $A^{\mathrm{d}}$ denoting the area of a single disk (in square pixels), and should be as close to the target value $\phi$ as possible. In addition, we impose the condition

$$
\frac{n^{\mathrm{d}}-\sum_{c=1}^{n^{\mathrm{c}}} n_{c}^{\mathrm{d}}}{(\ell-2 \rho)^{2}} \approx \frac{n^{\mathrm{t}} \sum_{c=1}^{n^{\mathrm{c}}} q_{c} n_{c}^{\mathrm{d}}}{2 \rho(\ell-\rho)}
$$

matching the local volume fractions of disks in interior and edge regions. Thus, given the numbers of disks attached to codes $\left\{n_{c}^{\mathrm{d}}\right\}_{c=1}^{n^{\mathrm{c}}}$, Eqs. (18) and (19) implicitly define 
tile edge lengths $\tilde{\ell}$ and $\hat{\ell}$, which should be equal to each other for the correct tile set setup. In our case, we sequentially check all values $\left\{n_{c}^{\mathrm{d}}\right\}_{c=1}^{n^{\mathrm{c}}}$ such that $n_{c}^{\mathrm{d}} \geq 0$, $\sum_{c=1}^{n^{c}} n_{c}^{\mathrm{d}} \leq n^{\mathrm{d}}$ and select the configuration with the minimum difference $|\tilde{\ell}-\hat{\ell}|^{*}{ }^{*}$

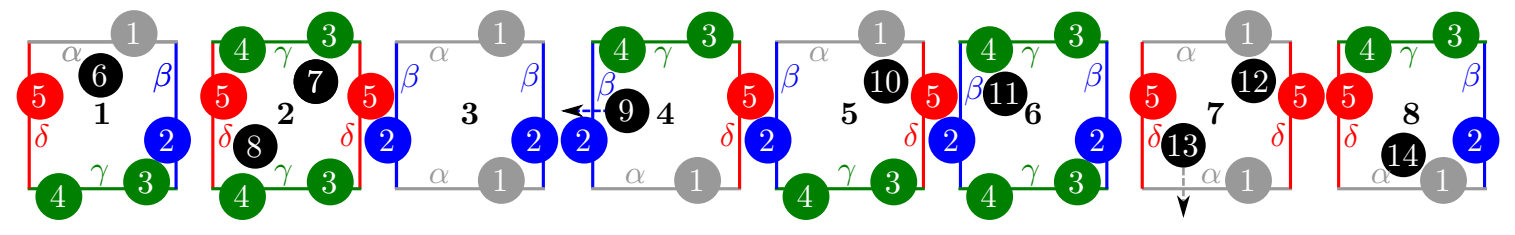

Figure 6. An example of an admissible $14\{1-1-2-1\}$ configuration (with $n_{\alpha}^{\mathrm{d}}=n_{\gamma}^{\mathrm{d}}=$ $n_{\delta}^{\mathrm{d}}=1$ and $n_{\beta}^{\mathrm{d}}=2$ code-related disks) and its modification by disk displacements; disk 9 leaves its parent tile 4 and randomly enters tiles $1,3,6$ or 8 ; disk 13 leaves its parent tile 7 and randomly enters tiles $1,3,5$ or 7 .

On the basis of these data, we randomly generate positions of individual disks and assign them to randomly selected tile interiors and edges, until an admissible configuration $\boldsymbol{p}$ is obtained. A single loop of the optimisation algorithm involves a sequential selection of a disk $d \in\left\{1, \ldots, n^{\mathrm{d}}\right\}$, and its movement given by

$$
\widehat{x}_{j, d}=x_{j, d}+\ell\left(U-\frac{1}{2}\right), \quad j=1,2,
$$

repeated until a new admissible configuration $\widehat{\boldsymbol{p}}$ is encountered. The symbol $U$ denotes a random variable with a uniform distribution in the interval $[0,1]$. If a disk, during its displacement, leaves its parent tile by crossing the edge of code $c$, it is randomly assigned to a tile sharing the same code, Figure 6.

The acceptance of the new solution $\widehat{\boldsymbol{p}}$ is driven by the Metropolis criterion [19]

$$
\exp \left(\frac{f(\boldsymbol{p})-f(\widehat{\boldsymbol{p}})}{\theta}\right) \geq U
$$

where $\theta$ denotes the algorithmic temperature, initially set to $\theta^{\max }$ and gradually reduced by a constant multiplicator $\theta^{\mathrm{mlt}}<1$ once the loop over all $n^{\mathrm{d}}$ disks is completed. The entire algorithm terminates after $n^{\max }$ objective function evaluations. Moreover, we keep it restarting when the current temperature $\theta$ is less than the threshold value $\theta^{\text {min }}$. Such a re-annealing step was found beneficial, as the resulting problem is multi-modal and discontinuous due to the presence of edge-constrained disks.

* Note that the values of $\ell$ and $n^{\mathrm{d}}$ are kept constant during the optimisation process, whereas the values of $n_{c}^{\mathrm{d}}$ are allowed to change, since disks can move freely between tile interiors and edges. 
(a) $10\{1-1-0-0\}, \widetilde{\phi}=23.6 \%$ ogirpyorax

(b) $17\{1-1-1-1\}, \widetilde{\phi}=27.9 \%$,

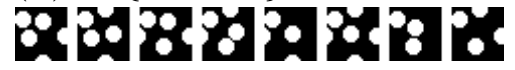

(c) $27\{2-1-1-1\}, \widetilde{\phi}=26.7 \%$

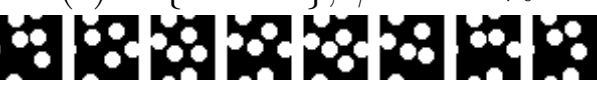

(d) $32\{2-1-2-1\}, \widetilde{\phi}=26.6 \%$
$10\{1-0-1-1\}, \widetilde{\phi}=28.0 \%$

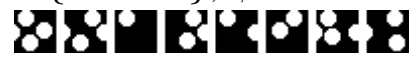

$17\{1-1-1-1\}, \widetilde{\phi}=27.9 \%$

Propopopto

$27\{2-1-1-1\}, \widetilde{\phi}=26.7 \%$

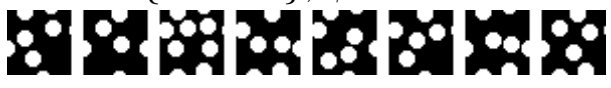

$32\{1-2-2-1\}, \widetilde{\phi}=26.6 \%$

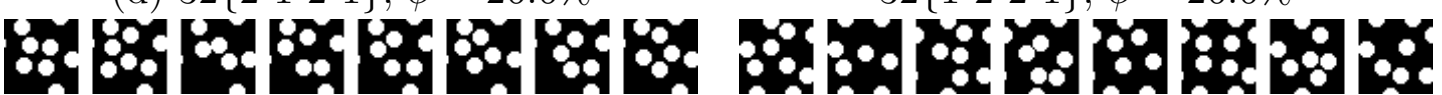

$$
w=10^{4}
$$ \\ $w=10^{5}$}

Figure 7. Optimised sets W8/2-2 obtained for weighting factors $w$ equal to $10^{4}$ and $10^{5}$ and for configurations with (a) $n^{\mathrm{d}}=10, \ell=42 \mathrm{px},(\mathrm{b}) n^{\mathrm{d}}=17$, $\ell=52 \mathrm{px},(\mathrm{c}) n^{\mathrm{d}}=27, \ell=64 \mathrm{px}$ and (d) $n^{\mathrm{d}}=38, \ell=74 \mathrm{px} ; n^{\mathrm{d}}\left\{n_{c}^{\mathrm{d}}\right\}_{c=1}^{n^{\mathrm{c}}}$ refers to configuration of $n^{\mathrm{d}}$ disks in total with $n_{c}^{\mathrm{d}}$ disks intersecting edge $c, \ell$ is the tile edge length and $\widetilde{\phi}$ is the reconstructed volume fraction.

periodic construction, e.g. [48]. In addition, their number and magnitude can be substantially reduced by increasing the edge length $\ell$, Figure $8(\mathrm{~b})$, and practically eliminated when using more general tile sets [33]. For lower values of $w$, the disk distribution becomes more regular, Figure 8(c), and the resulting representation is visually indistinguishable from the periodic setting, cf. [33].

Such conclusions are further supported by Figure 9 showing cross-sections of the two-point probability functions in the $k_{1}$ direction for two different values of the weighting factor $w$. The results demonstrate that for higher values of $w$, the short-range phenomena are captured to a high accuracy and the magnitude of local extremes are consistently reduced with the increasing number of disks, albeit at a small rate. By decreasing the emphasis on $S_{2}$ objective, Figure $9(\mathrm{~b})$, the discrepancy between the original and reconstructed medium substantially increases at short distances, leading even to an inconsistent value of the volume fraction for 10 disks. The local peaks also become more pronounced as the stress-based criterion drives the system towards periodic configurations.

Figure 10(a) illustrates the ability of the optimisation algorithm to achieve selfequlibrated stress enrichment functions by comparing the distribution of tractions $T_{1}^{*(3)}$ obtained for an initial and the optimised configuration of disks. Clearly, traction 
(a)
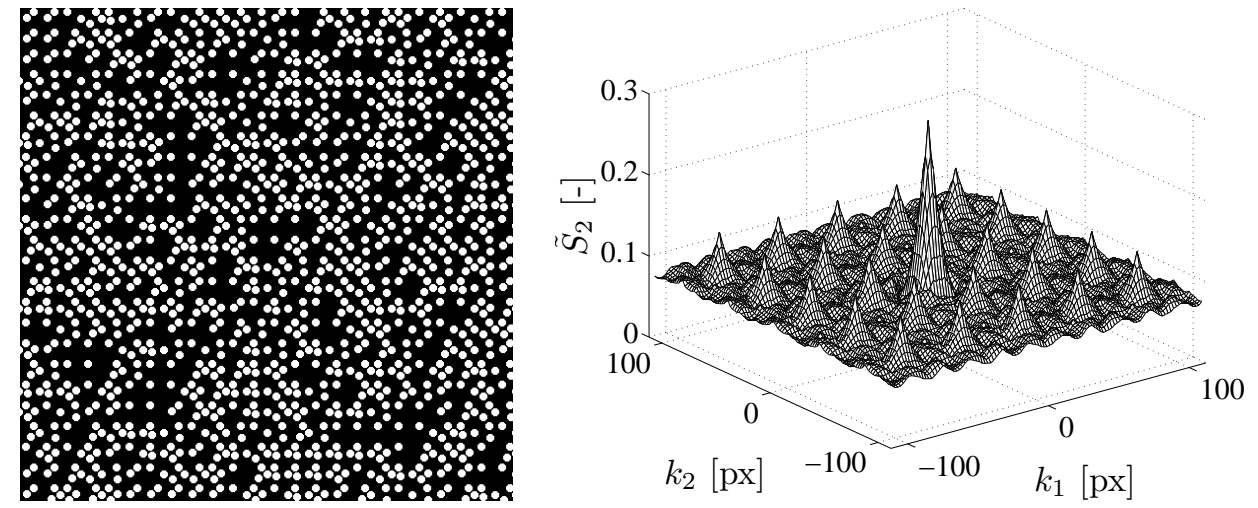

(b)
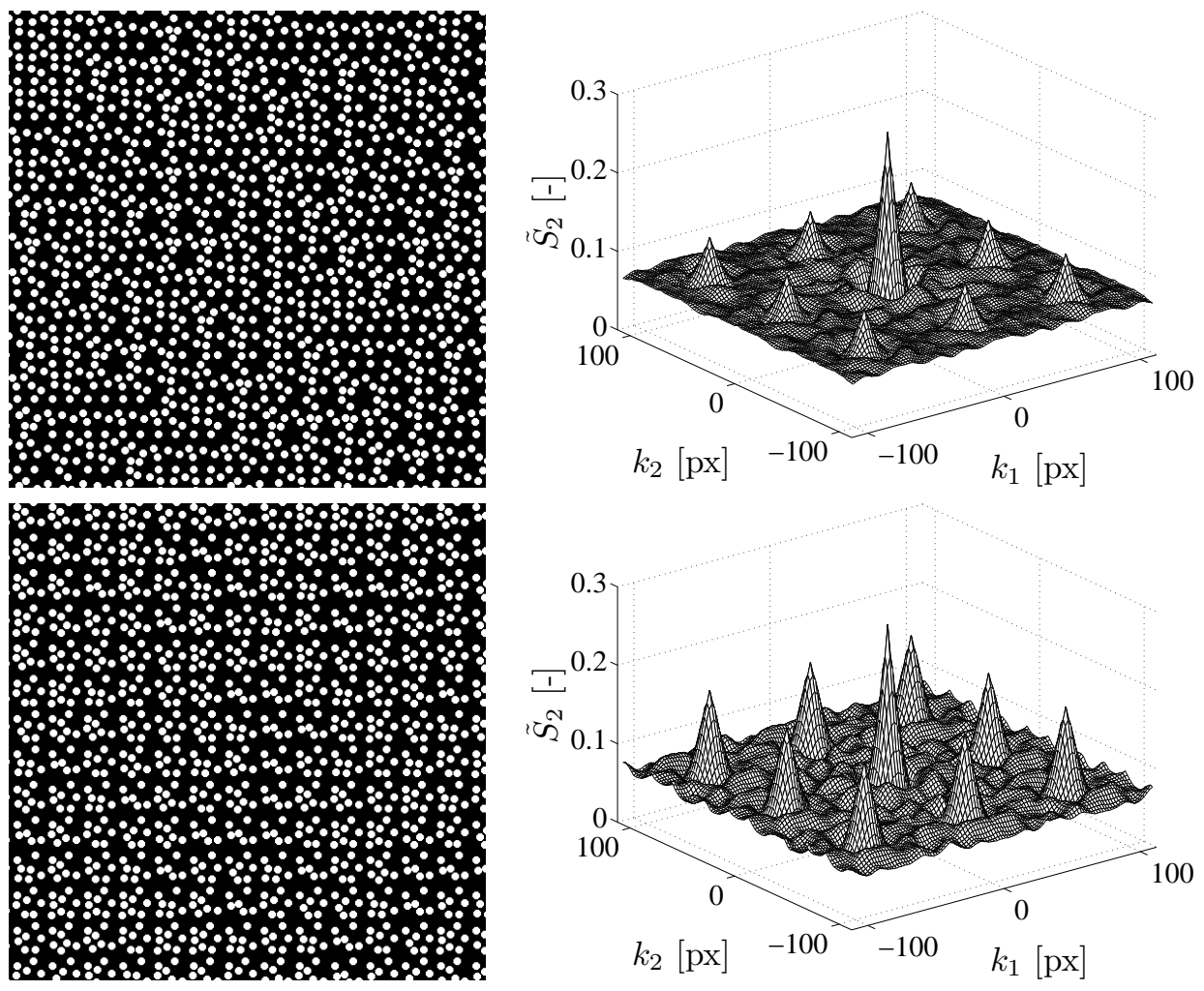

(c)

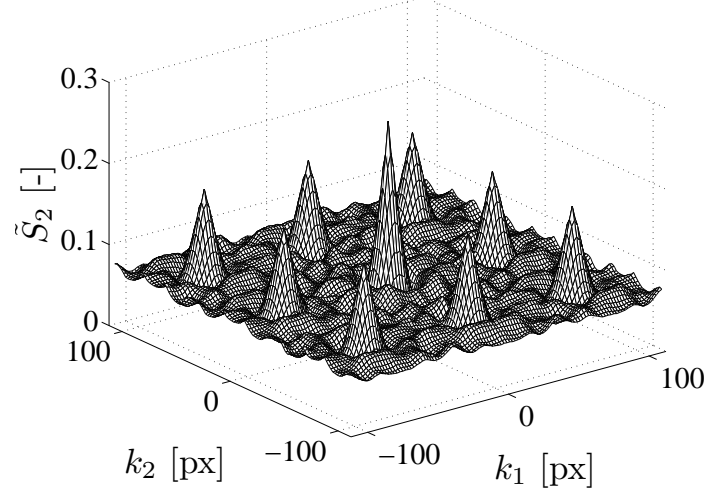

Figure 8. Reconstructed microstructures and two point probability functions $\widetilde{S}_{2}$ for tile sets with (a) $w=10^{5}, n^{\mathrm{d}}=10$ disks and $\ell=42 \mathrm{px}$, (b) $w=10^{5}, n^{\mathrm{d}}=38$ disks and $\ell=74 \mathrm{px}$, and (c) $w=10^{4}, n^{\mathrm{d}}=38$ disks and $\ell=74 \mathrm{px}$.

enrichments at contiguous edges differ significantly in the initial configuration, and are reduced to almost identical values by the proposed procedure. This also 
(a)

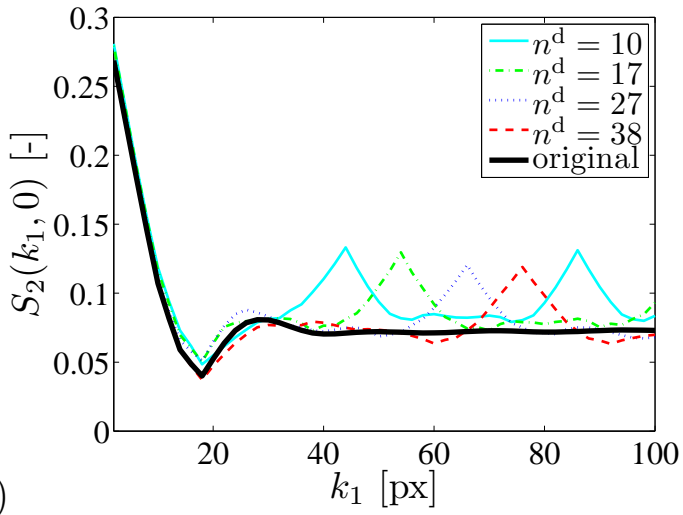

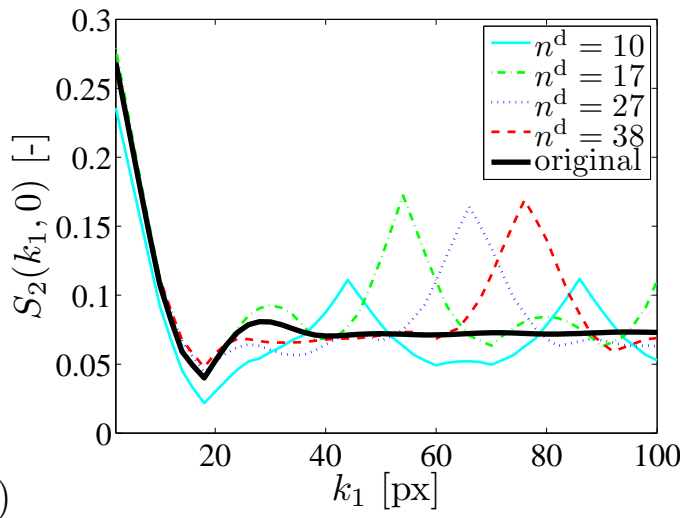

(b)

Figure 9. Comparison of two-point probability functions $S_{2}\left(k_{1}, 0\right)$ for the weighting factors (a) $w=10^{5}$ and (b) $w=10^{4}$.

automatically keeps the edge jumps in reconstructed traction enrichments $\left[\widetilde{T}_{1}^{*(3)}\right]$ under control, Figure 10(b), since their magnitude corresponds to the scatter found for representative eight tiles from $\mathcal{O}_{\mathrm{T}}$ utilised in the reconstruction, recall Figure $4(\mathrm{~b})$.

(a)
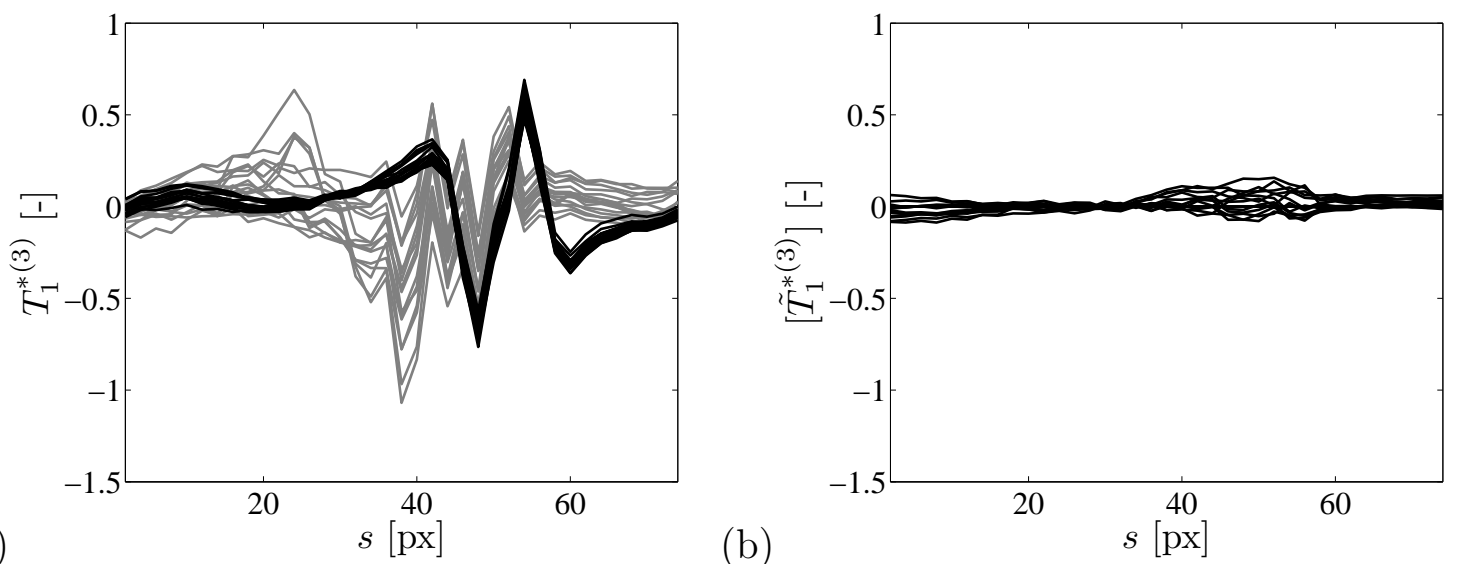

Figure 10. Distribution of (a) traction enrichments $T_{1}^{*(3)}$ and (b) reconstructed traction enrichment jumps $\left[\widetilde{T}_{1}^{*(3)}\right]$ at edges $\Gamma_{\delta}$ of the tiling $\mathcal{O}_{\mathrm{T}}$, obtained for $n^{\mathrm{d}}=38$ disks and $\ell=74 \mathrm{px}$. The grey/black patterns in (a) correspond to initial and optimised and enrichment functions, respectively.

To what extent influences such choice of representative tiles the synthesised enrichment functions? To address this question, we consider a particular 
reconstruction of stress enrichments functions $\widetilde{\Sigma}^{*}: \mathcal{O}_{\mathrm{T}} \rightarrow \mathbb{R}^{3 \times 3}$, assembled according to the the sequence of tiles found in the tiling $\mathcal{O}_{\mathrm{T}}$. It is useful for the visualisation purposes to introduce a local error measure

$$
f_{i j}^{\Sigma}(\boldsymbol{k})=\frac{\left|\Sigma_{i j}^{*}(\boldsymbol{k})-\widetilde{\Sigma}_{i j}^{*}(\boldsymbol{k})\right|}{\max _{\boldsymbol{m} \in \mathbb{K}^{\mathcal{O}_{\mathrm{T}}}} \Sigma_{i j}^{*}(\boldsymbol{m})-\min _{\boldsymbol{m} \in \mathbb{K}^{\mathcal{O}_{\mathrm{T}}}} \Sigma_{i j}^{*}(\boldsymbol{m})}, \quad \boldsymbol{k} \in \mathbb{K}^{\mathcal{O}_{\mathrm{T}}} ; i, j \in\{1,2,3\},
$$

quantifying a difference between the components of the stress enrichment functions $\Sigma^{*}$ determined directly for the tiling $\mathcal{O}_{\mathrm{T}}$ by the algorithm described in Appendix A, and their reconstruction $\widetilde{\Sigma}^{*}$.
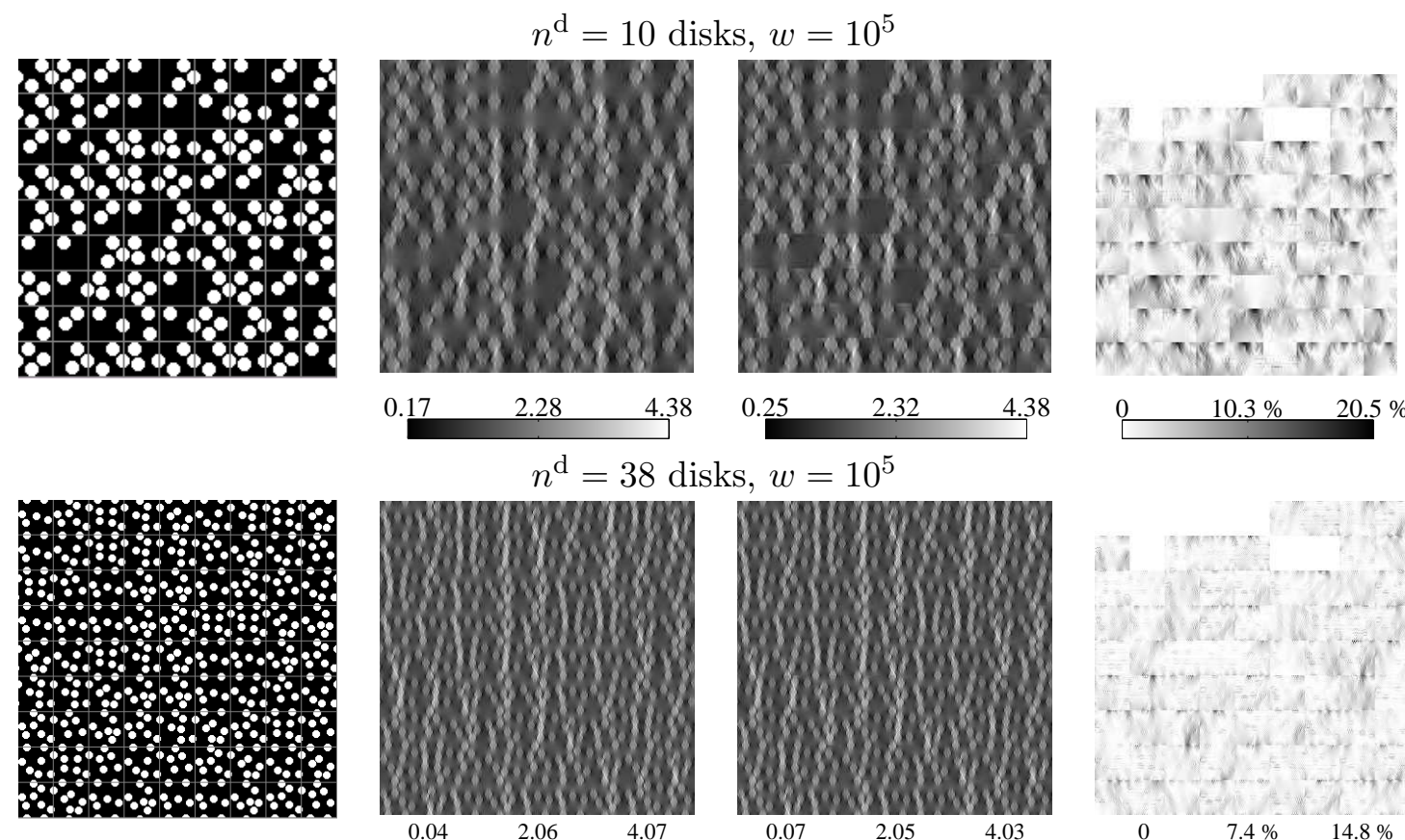

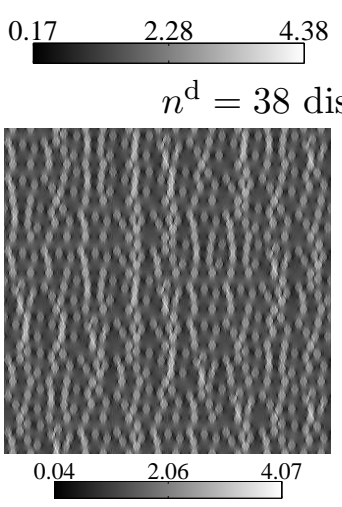

(b)
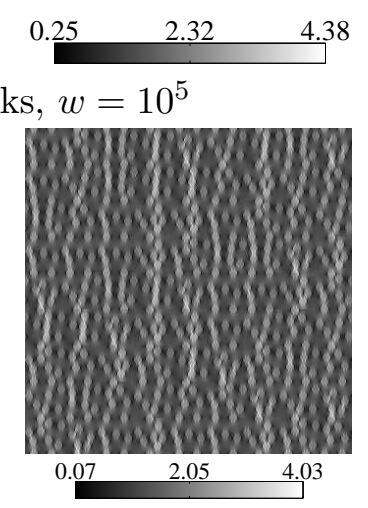

(c)
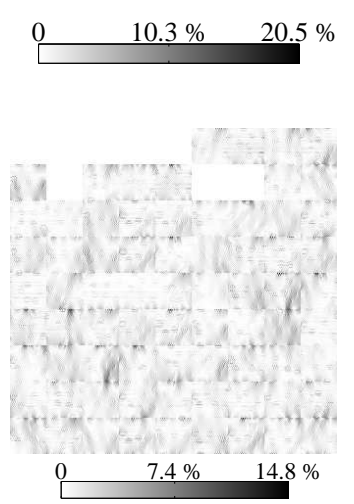

(d)

Figure 11. Assessment of tiling-based enrichment functions, (a) microstructures obtained by tilings $\mathcal{O}_{\mathrm{T}}$, distribution of (b) true stress enrichment functions $\Sigma_{13}^{*} \equiv$ $\sigma_{11}^{*(3)}$, (c) reconstructed stress enrichment functions $\widetilde{\Sigma}_{13}^{*} \equiv \widetilde{\sigma}_{11}^{*(3)}$ and of $(\mathrm{d})$ the local reconstruction-based error $f_{13}^{\Sigma}$.

Outcomes of this comparison are shown in Figure 11 in the form of (a) tilingbased microstructures, (b) distribution of the corresponding enrichment functions 
$\Sigma_{13}^{*}$, (c) their reconstructed counterparts and (d) spatial distribution of the relative error. For the microstructure generated from tiles with $n^{\mathrm{d}}=10$ disks, we observe that the reconstructed field displays distributed errors in tile interiors. Similarly to $S_{2}$ criterion, these deviations are significantly reduced and become highly localised when increasing the number of disks and the size of tiles. This claim is further supported by Figure 12, plotting the evolution of the global error

$$
f^{\Sigma}=\frac{1}{\mathcal{O}_{\mathrm{T}}} \sum_{i, j=1}^{3} \sum_{\boldsymbol{k} \in \mathbb{K}^{\mathcal{O}_{\mathrm{T}}}}\left|f_{i j}^{\Sigma}(\boldsymbol{k})\right|
$$

as a function of the number of disks. For both values of $w$, we observe approximately linear convergence with increasing $n^{\mathrm{d}}$. In addition, the error decreases for larger phase contrasts $E^{\mathrm{d}} / E^{\mathrm{m}}$. This is caused by the fact that stresses tend to concentrate more at stiffer disks, therefore reducing variations of tractions at tile edges, see also [32] for a similar discussion.

(a)

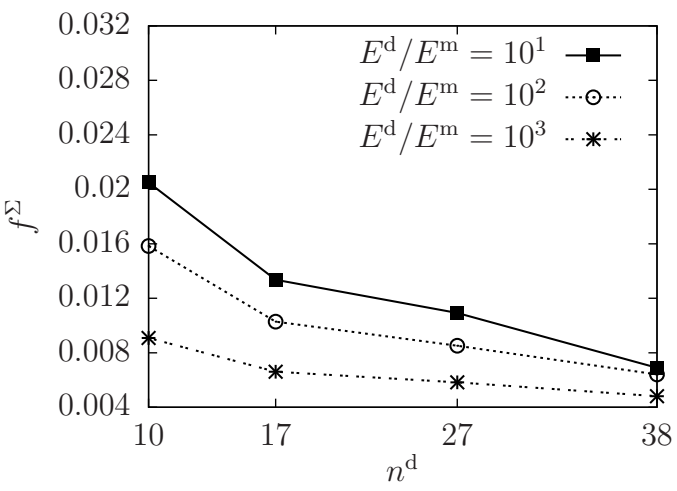

Figure 12. The global reconstruction error $f^{\Sigma}$ as a function of the number of disks $n^{\mathrm{d}}$ for different phase contrasts $E^{\mathrm{d}} / E^{\mathrm{m}}$ and weighting factors (a) $w=10^{5}$ and (b) $w=10^{4}$.

Altogether, this indicates that the tile set was designed correctly, since the optimisation was executed for independent objective functions, recall Equation (17). Finally we stress that the significant compression has been achieved by the tilingbased representation: the original microstructure contains $\approx 1,300$ disks, whereas the most detailed tile-based representation builds on 38 disks only and is capable of producing much larger microstructures at a negligible computational cost. 


\section{Conclusions}

In this work, we have proposed an approach to the construction of aperiodic local fields in heterogeneous media with potential applications in hybrid or generalised FE environments. The method is based on the Wang tiling concept that allows us to represent complex patterns using a limited set of representative tiles, complemented by the Simulated Annealing-based algorithm to arrive at optimal tile set morphologies. On the basis of the results obtained from analyses of the medium under consideration we conjecture that:

- the proposed method provides a robust tool for compression of disordered microstructures and can serve as an efficient microstructure generation algorithm,

- it allows for aperiodic extensions of local, possibly periodic, fields to substantially larger domains while maintaining their compatibility,

- the tiling-based fields can be utilised as microstructure-based enrichment functions for generalised Partition of Unity methods or hybrid finite element schemes.

We are fully aware that our conclusions are somewhat provisional, in the sense that these are based on a single set of tiles and the specific class of microstructures. Partial extension to general setting is available in $[33,7]$ and remains in the focus of our current work.

Acknowledgements The authors thank Jaroslav Vondřejc (CTU in Prague) for providing us with a MATLAB source code of FFT-based homogenisation algorithm and Adrian Russell (University of New South Wales), Michal Šejnoha and Milan Jirásek (CTU in Prague) and anonymous referees for their criticism and helpful comments on earlier versions of the manuscript. We also gratefully acknowledge financial support by the Czech Science Foundation through grants No. P105/12/0331 (JN), P105/11/P370 (AK), and P105/11/0411 (JZ). Our work was partially supported by the European Social Fund, grant No. CZ.1.07/2.3.00/30.0005

of Brno University of Technology (Support for the creation of excellent interdisciplinary research teams at Brno University of Technology, JN), by the Ministry of Education, Youth and Sports of the Czech Republic through project 
MSM 6840770003 (AK), and by the European Regional Development Fund under the IT4Innovations Centre of Excellence, project No. CZ.1.05/1.1.00/02.0070 (JZ).

\section{Appendix A. Computation of mechanical fields}

As explained earlier in Section 1, our objective is to determine local fields within a given domain $\mathcal{Y} \subset \mathbb{R}^{2}$ subjected to a given overall strain field

$$
\boldsymbol{E}=\left[\begin{array}{lll}
E_{11} & E_{22} & \sqrt{2} E_{12}
\end{array}\right]^{\top},
$$

under the periodic boundary conditions. These follow from the solution of the elastic unit cell problem $[28,27]$

$$
\boldsymbol{\varepsilon}(\boldsymbol{x})=\boldsymbol{\partial u}(\boldsymbol{x}), \boldsymbol{\partial}^{\top} \boldsymbol{\sigma}(\boldsymbol{x})=\mathbf{0}, \boldsymbol{\sigma}(\boldsymbol{x})=\boldsymbol{L}(\boldsymbol{x}) \boldsymbol{\varepsilon}(\boldsymbol{x}) \text { for } \boldsymbol{x} \in \mathcal{Y},
$$

in which $\boldsymbol{u}: \mathcal{Y} \rightarrow \mathbb{R}^{2}$ designates the displacement field, $\boldsymbol{\varepsilon}: \mathcal{Y} \rightarrow \mathbb{R}^{3}$ and $\boldsymbol{\sigma}: \mathcal{Y} \rightarrow \mathbb{R}^{3}$ denote the $\mathcal{Y}$-periodic strain and stress fields, $\boldsymbol{L}: \mathcal{Y} \rightarrow \mathbb{R}^{3 \times 3}$ stands for the symmetric positive-definite material stiffness matrix, and the operator matrix is defined as

$$
\boldsymbol{\partial}=\left[\begin{array}{ccc}
\frac{\partial}{\partial x_{1}} & 0 & \frac{1}{\sqrt{2}} \frac{\partial}{\partial x_{2}} \\
0 & \frac{\partial}{\partial x_{2}} & \frac{1}{\sqrt{2}} \frac{\partial}{\partial x_{1}}
\end{array}\right]^{\top} .
$$

In addition, the strain field is subject to a mean value-type constraint

$$
\frac{1}{|\mathcal{Y}|} \int_{\mathcal{Y}} \varepsilon(\boldsymbol{x}) \mathrm{d} \boldsymbol{x}=\boldsymbol{E}
$$

It is well-known $[27,28]$ that the solution to the unit cell is characterised by the Lippmann-Schwinger equation

$$
\varepsilon(\boldsymbol{x})+\int_{\mathcal{Y}} \boldsymbol{\Gamma}^{0}(\boldsymbol{x}-\boldsymbol{y}) \delta \boldsymbol{L}(\boldsymbol{y}) \varepsilon(\boldsymbol{y}) \mathrm{d} \boldsymbol{y}=\boldsymbol{E} \text { for } \boldsymbol{x} \in \mathcal{Y},
$$

where $\delta \boldsymbol{L}=\boldsymbol{L}-\boldsymbol{L}^{0}, \boldsymbol{L}^{0} \in \mathbb{R}^{3 \times 3}$ is the stiffness matrix of an auxiliary reference medium and the operator $\Gamma^{0}: \mathcal{Y} \rightarrow \mathbb{R}^{3 \times 3}$ is related to the Green function of the problem (A.2) with $\boldsymbol{L}(\boldsymbol{x})=\boldsymbol{L}^{0}$. It admits a compact closed-form expression in the Fourier space, e.g. [27, Section 5.3], and its action can be efficiently evaluated by the FFT algorithm. This observation is at the heart of an iterative scheme due to Moulinec and Suquet [30], which can be applied to arbitrary digitised media. 
In our case, we adopt an accelerated version of the original algorithm based on observations due to Zeman et al. [47]. Since the sample is discretized by a regular $n_{1}^{\mathcal{Y}} \times n_{2}^{\mathcal{y}}$ bitmap, it is convenient to project the integral equation onto the space of trigonometric polynomials, e.g. [39]. This yields the linear system in the form

$$
(\boldsymbol{I}+\boldsymbol{B}) \boldsymbol{e}=\boldsymbol{b},
$$

where $\boldsymbol{e} \in \mathbb{R}^{3 \times n_{1}^{y} \times n_{2}^{y}}$ stores the unknown strain values at individual pixels, $\boldsymbol{b} \in$ $\mathbb{R}^{3 \times n_{1}^{y} \times n_{2}^{y}}$ is the corresponding matrix of overall strains and matrix $\boldsymbol{B}$ is expressed as a product of several matrices

$$
\begin{aligned}
\boldsymbol{B} & =\left(\begin{array}{ccc}
\boldsymbol{F}^{-1} & \mathbf{0} & \mathbf{0} \\
\mathbf{0} & \boldsymbol{F}^{-1} & \mathbf{0} \\
\mathbf{0} & \mathbf{0} & \boldsymbol{F}^{-1}
\end{array}\right)\left(\begin{array}{ccc}
\boldsymbol{\Gamma}_{1111} & \boldsymbol{\Gamma}_{1122} & \sqrt{2} \boldsymbol{\Gamma}_{1112} \\
\boldsymbol{\Gamma}_{2211} & \boldsymbol{\Gamma}_{2222} & \sqrt{2} \boldsymbol{\Gamma}_{1112} \\
\sqrt{2} \boldsymbol{\Gamma}_{2212} & \sqrt{2} \boldsymbol{\Gamma}_{1212} & 2 \boldsymbol{\Gamma}_{2212}
\end{array}\right)\left(\begin{array}{ccc}
\boldsymbol{F} & \mathbf{0} & \mathbf{0} \\
\mathbf{0} & \boldsymbol{F} & \mathbf{0} \\
\mathbf{0} & \mathbf{0} & \boldsymbol{F}
\end{array}\right) \\
& \times\left(\begin{array}{ccc}
\delta \boldsymbol{L}_{1111} & \delta \boldsymbol{L}_{1122} & \sqrt{2} \delta \boldsymbol{L}_{1112} \\
\delta \boldsymbol{L}_{2211} & \delta \boldsymbol{L}_{2222} & \sqrt{2} \delta \boldsymbol{L}_{1112} \\
\sqrt{2} \delta \boldsymbol{L}_{2212} & \sqrt{2} \delta \boldsymbol{L}_{1222} & 2 \delta \boldsymbol{L}_{2212}
\end{array}\right) .
\end{aligned}
$$

Here, $\boldsymbol{F} \in \mathbb{C}^{n_{1}^{y} \times n_{2}^{y}}$ and $\boldsymbol{F}^{-1}$ implement the forward and the inverse Fourier transform and, e.g., $\delta \boldsymbol{L}_{1122} \in \mathbb{R}^{n_{1}^{\mathcal{y}} \times n_{2}^{\nu}}$ stores the corresponding component of the stiffness tensor at individual pixels, see [47] for more details. The system (A.6) is solved using standard conjugate gradient algorithm. Upon convergence, the distribution of the local stress field $\boldsymbol{\sigma}$ is determined from the solution $\boldsymbol{e}$ by Equation (A.2) 3 $_{3}$ The local displacement fields $\boldsymbol{u}$ follow from an inexpensive analysis in the Fourier space, e.g. [45].

Note that the construction of the enrichment functions is based on the perturbation fields of displacements and stresses

$$
\begin{aligned}
& \boldsymbol{u}^{*}(\boldsymbol{x})=\boldsymbol{u}(\boldsymbol{x})-\frac{1}{|\mathcal{Y}|} \int_{\mathcal{Y}} \boldsymbol{u}(\boldsymbol{y}) \mathrm{d} \boldsymbol{y}, \\
& \boldsymbol{\sigma}^{*}(\boldsymbol{x})=\boldsymbol{\sigma}(\boldsymbol{x})-\frac{1}{|\mathcal{Y}|} \int_{\mathcal{Y}} \boldsymbol{\sigma}(\boldsymbol{y}) \mathrm{d} \boldsymbol{y},
\end{aligned}
$$

instead of the total values. The enrichment functions for displacements, $\Psi^{*}$ in Equation (2), and stresses, $\boldsymbol{\Sigma}^{*}$ in Equation (6), can now be constructed from the solutions to three load-cases, obtained by successively setting each component of $E_{i j}$ in (A.1) to 1, while the ones become 0 . 
[1] I. Babuška and R. Lipton. Optimal local approximation spaces for generalized finite element methods with application to multiscale problems. Multiscale Modeling and Simulation, 9(1):373-406, 2011, 1004.3041.

[2] T. Belytschko, R. Gracie, and G. Ventura. A review of extended/generalized finite element methods for material modeling. Modelling and Simulation in Materials Science and Engineering, 17(4):043001, 2009.

[3] M.F. Cohen, J. Shade, S. Hiller, and O. Deussen. Wang tiles for image and texture generation. ACM Transactions on Graphics, 22(3):287-294, 2003.

[4] K. Culik. An aperiodic set of 13 Wang tiles. Discrete Mathematics, 160:245-251, 1996.

[5] K. Culik and J. Kari. An aperiodic set of Wang cubes. Journal of Universal Computer Science, 1(10):675-686, 1995.

[6] E.D. Demaine and M.L. Demaine. Jigsaw puzzles, edge matching, and polyomino packing: Connections and complexity. Graphs and Combinatorics, 23:195-208, 2007.

[7] M. Doškář. Geometrical modelling of heterogenous materials based on Wang tilings. Bachelor thesis, Czech Technical University in Prague, 2012. Available at http://mech.fsv.cvut. cz/wiki/index.php/File:BP-2012-Doskar.pdf (in Czech).

[8] J.D. Eshelby. The determination of the elastic field of an ellipsoidal inclusion, and related problems. Proceedings of the Royal Society of London. Series A, Mathematical and Physical Sciences, 241(1226):376-396, 1957.

[9] J.D. Eshelby. The elastic field outside an ellipsoidal inclusion. Proceedings of the Royal Society of London. Series A. Mathematical and Physical Sciences, 252(1271):561-569, 1959.

[10] F. Feyel and J.-L. Chaboche. FE ${ }^{2}$ multiscale approach for modelling the elastoviscoplastic behaviour of long fibre $\mathrm{SiC} / \mathrm{Ti}$ composite materials. Computer Methods in Applied Mechanics and Engineering, 183(3-4):309-330, 2000.

[11] J. Fish and Z. Yuan. Multiscale enrichment based on partition of unity. International Journal for Numerical Methods in Engineering, 62(10):1341-1359, 2005.

[12] J. Fish and Z. Yuan. Multiscale enrichment based on partition of unity for nonperiodic fields and nonlinear problems. Computational Mechanics, 40:249-259, 2007.

[13] T.-P. Fries and T. Belytschko. The extended/generalized finite element method: An overview of the method and its applications. International Journal for Numerical Methods in Engineering, 84(3):253-304, 2010.

[14] J. Gajdošík, J. Zeman, and M. Šejnoha. Qualitative analysis of fiber composite microstructure: Influence of boundary conditions. Probabilistic Engineering Mechanics, 21(4):317-329, 2006.

[15] M.G.D. Geers, V.G. Kouznetsova, and W.A.M. Brekelmans. Multi-scale computational homogenization: Trends and challenges. Journal of Computational and Applied Mathematics, 234:2175-2182, 2010 .

[16] A. Glassner. Andrew Glassner's notebook. Computer Graphics and Applications, IEEE, 24(1):86-91, 2004.

[17] I. Herrera. Trefftz method: a general theory. Numerical Methods for Partial Differential Equations, 16(6):561-580, 2000.

[18] Ł. Kaczmarczyk and C.J. Pearce. A corotational hybrid-Trefftz stress formulation for modelling cohesive cracks. Computer Methods in Applied Mechanics and Engineering, 198(15-16):1298- 
1310, 2009 .

[19] S. Kirkpatrick, C. Jr. Gelatt, and M. P. Vecchi. Optimization by simulated annealing. Science, 220:671-680, 1983.

[20] H. Kumar, C.L. Briant, and W.A. Curtin. Using microstructure reconstruction to model mechanical behavior in complex microstructures. Mechanics of Materials, 38(8-10):818-832, 2006.

[21] A. Lagae and P. Dutré. A comparison of methods for generating Poisson disk distributions. Computer Graphics Forum, 27(1):114-129, 2008.

[22] H. Lee, M. Brandyberry, A. Tudor, and K. Matouš. Three-dimensional reconstruction of statistically optimal unit cells of polydisperse particulate composites from microtomography. Physical Review E, 80:061301, 2009.

[23] M. Lepš. Single and Multi-Objective Optimization in Civil Engineering with Applications. $\mathrm{PhD}$ thesis, CTU in Prague, 2005. Available at http://klobouk.fsv.cvut.cz/ leps/ publications/pdf/thesis.pdf.

[24] A. Lu, D.S. Ebert, W. Qiao, M. Kraus, and B. Mora. Volume illustration using Wang cubes. ACM Transactions on Graphics, 26, 2007.

[25] A.M. Matache, I. Babuška, and C. Schwab. Generalized p-FEM in homogenization. Numerische Mathematik, 86:319-375, 2000.

[26] J.M. Melenk and I. Babuška. The partition of unity finite element method: Basic theory and applications. Computer Methods in Applied Mechanics and Engineering, 139(1-4):289-314, 1996.

[27] J.C. Michel, H. Moulinec, and P. Suquet. Effective properties of composite materials with periodic microstructure: a computational approach. Computer Methods in Applied Mechanics and Engineering, 172(1-4):109 - 143, 1999.

[28] G. W. Milton. The Theory of Composites, volume 6 of Cambridge Monographs on Applied and Computational Mathematics. Cambridge University Press, 2002.

[29] R. Morgan and I. Babuška. An approach for constructing families of homogenized equations for periodic media. I: An integral representation and its consequences. SIAM Journal on Mathematical Analysis, 22(1):1-15, 1991.

[30] H. Moulinec and P. Suquet. A fast numerical method for computing the linear and nonlinear mechanical properties of composites. Comptes rendus de l'Académie des sciences. Série II, Mécanique, physique, chimie, astronomie, 318(11):1417-1423, 1994.

[31] S.R. Niezgoda, D.M. Turner, D.T. Fullwood, and S.R. Kalidindi. Optimized structure based representative volume element sets reflecting the ensemble-averaged 2-point statistics. Acta Materialia, 58(13):4432-4445, 2010.

[32] J. Novák, Ł. Kaczmarczyk, P. Grassl, J. Zeman, and C. Pearce. A micromechanics-enhanced finite element formulation for modelling heterogeneous materials. Computer Methods in Applied Mechanics and Engineering, 201-204:53-64, 2012, 1103.5633.

[33] J. Novák, A. Kučerová, and J. Zeman. Compressing random microstructures via stochastic Wang tilings. Physical Review E, 86:040104, 2012, 1206.0933.

[34] J.T. Oden, T. Belytschko, J. Fish, T.J.R. Hughes, C. Johnson, D. Keyes, A. Laub, L. Petzold, D. Srolovitz, and S. Yip. Simulation-based engineering science: Revolutionizing engineering 
science through simulation. Report of the National Science Foundation blue ribbon panel on simulation-based engineering science, National Science Foundation, Arlington, VA, 2006. Available at http://www.nsf.gov/pubs/reports/sbes_final_report.pdf.

[35] B. Pichler, S. Scheiner, and C. Hellmich. From micron-sized needle-shaped hydrates to meter-sized shotcrete tunnel shells: Micromechanical upscaling of stiffness and strength of hydrating shotcrete. Acta Geotechnica, 3(4):273-294, 2008.

[36] G.L. Povirk. Incorporation of microstructural information into models of two-phase materials. Acta Metallurgica et Materialia, 43(8):3199-3206, 1995.

[37] F.K.F. Radtke, A. Simone, and L.J. Sluys. A partition of unity finite element method for obtaining elastic properties of continua with embedded thin fibres. International Journal for Numerical Methods in Engineering, 84(6):708-732, 2010.

[38] F.K.F. Radtke, A. Simone, and L.J. Sluys. A partition of unity finite element method for simulating non-linear debonding and matrix failure in thin fibre composites. International Journal for Numerical Methods in Engineering, 86(4-5):453-476, 2011.

[39] J. Saranen and G. Vainikko. Periodic Integral and Pseudodifferential Equations with Numerical Approximation. Springer Monographs in Mathematics. Springer-Verlag, Berlin, Heidelberg, 2002.

[40] J. Schröder, D. Balzani, and D. Brands. Approximation of random microstructures by periodic statistically similar representative volume elements based on lineal-path functions. Archive of Applied Mechanics, 81:975-997, 2011.

[41] J.A. Teixeira de Freitas. Formulation of elastostatic hybrid-Trefftz stress elements. Computer Methods in Applied Mechanics and Engineering, 153:127-151, 1998.

[42] S. Torquato. Random heterogenous materials. Springer-Verlag, New York, 2002.

[43] J. Cerný. Thermodynamical approach to the traveling salesman problem: An efficient simulation algorithm. Journal of Optimization Theory and Applications, 45:41-51, 1985.

[44] H. Wang. Proving theorems by pattern recognition-II. Bell Systems Technical Journal, 40(2):141, 1961.

[45] F. Willot, Y.-P. Pellegrini, M.I. Idiart, and P.P. Castañeda. Effective-medium theory for infinite-contrast two-dimensionally periodic linear composites with strongly anisotropic matrix behavior: Dilute limit and crossover behavior. Physical Review B, 78:104111, 2008, 0804.2817.

[46] C.L.Y. Yeong and S. Torquato. Reconstructing random media. Physical Review E, 57(1):495506, 1998.

[47] J. Zeman, J. Vondřejc, J. Novák, and I. Marek. Accelerating a FFT-based solver for numerical homogenization of periodic media by conjugate gradients. Journal of Computational Physics, 229(21):8065-8071, 2010, 1004.1122.

[48] J. Zeman and M. Šejnoha. From random microstructures to representative volume elements. Modelling and Simulation in Materials Science and Engineering, 15(4):S325-S335, 2007. 2007 Higlight paper. 